\title{
Acute cardiac injury in adult hospitalized COVID-19 patients in Zhuhai, China
}

\author{
Lizi Jin ${ }^{1 \#}$, Wenyi Tang ${ }^{1 \#}$, Lizheng Song ${ }^{1 \#}$, Liyun Luo ${ }^{1 \#}$, Zhijuan Zhou ${ }^{1}$, Xiuwu Fan ${ }^{1}$, Jinyou Zhang ${ }^{1}$, \\ Niujian $W^{1}{ }^{1}$, Kan Liu $^{2}$, Jian Chen ${ }^{1}$ \\ ${ }^{1}$ Department of Cardiovascular Medicine, Fifth Affiliated Hospital of Sun Yat-sen University, Zhuhai, China; ${ }^{2}$ Division of Cardiology, University of \\ Iowa, Iowa City, IA 52240, USA \\ Contributions: (I) Conception and design: J Chen, K Liu; (II) Administrative support: J Chen; (III) Provision of study materials or patients: J Chen; (IV) \\ Collection and assembly of data: L Song, L Luo, Z Zhou; (V) Data analysis and interpretation: L Jin, W Tang, J Chen; (VI) Manuscript writing: All \\ authors; (VII) Final approval of manuscript: All authors. \\ \#These authors contributed equally to this work. \\ Correspondence to: Jian Chen, MD. Department of Cardiovascular Medicine, Fifth Affiliated Hospital of Sun Yat-sen University, No.52 Meihua East \\ Rd. Zhuhai 519000, China. Email: chenjn@mail.sysu.edu.cn; Kan Liu, MD, PhD. Division of Cardiology, University of Iowa, Iowa City, IA 52240, \\ USA. Email: kan-liu@uiowa.edu.
}

Background: Coronavirus disease 2019 (COVID-19) has already became a public health emergency of international concern. COVID-19 related cardiac injury remains largely unclear.

Methods: We retrospectively analyzed demographic, clinical, laboratory and cardiovascular imaging data of all consecutively admitted adult COVID-19 patients in Zhuhai, China from January 17th, 2020 to February 18th, 2020.

Results: A total of 93 patients were included in the study. Acute cardiac injury was found in 9 (9.7\%) COVID-19 patients with median level of hypersensitive cardiac troponin I (hs-cTnI) to be $0.085 \mu \mathrm{g} / \mathrm{L}$ (IQR $0.027-0.560 \mu \mathrm{g} / \mathrm{L})$. Compared with patients without cardiac injury, the median age of patients with cardiac injury was significantly older (65.0 vs. 44.0, $\mathrm{P}<0.05)$, hypertension was significantly more common $(44.4 \%$ vs. $14.3 \%, \mathrm{P}<0.05)$, and the proportion of severe-critical cases were greater $(77.8 \%$ vs. $17.9 \%, \mathrm{P}<0.05)$. Patients with cardiac injury were more likely have elevation of N-terminal proBNP (NT-proBNP) in comparison (66.7\% vs. $10.0 \%, \mathrm{P}<0.05$ ). There was no significant difference in echocardiographic parameters between patients with and without cardiac injury. Multivariable logistic regression analysis indicated that older age (OR: 1.093, 95\% CI: 1.011-1.182) and increased NT-proBNP (OR: 10.979, 95\% CI: 2.024-59.555) were independent risk factors for cardiac injury. Cardiac magnetic resonance (CMR) imaging performed on three patients at around one month after they underwent significant hs-cTnI elevation showed that they had underlying cardiovascular comorbidities.

Conclusions: Acute cardiac injury was seen in the minority of hospitalized COVID-19 patients in Zhuhai, China. Older age and increased NT-proBNP were associated with acute cardiac injury.

Registration number: ChiCTR2000030952

Keywords: Severe acute respiratory syndrome coronavirus 2 (SARS-CoV-2); coronavirus disease 2019 (COVID-19); acute cardiac injury; cardiac magnetic resonance imaging

Submitted Jul 05, 2020. Accepted for publication Sep 10, 2020.

doi: $10.21037 / \mathrm{cdt}-20-607$

View this article at: http://dx.doi.org/10.21037/cdt-20-607 


\section{Introduction}

The outbreak of epidemic pneumonia caused by severe acute respiratory syndrome coronavirus 2 (SARS-CoV-2), which was later named as coronavirus disease 2019 (COVID-19), has generated multiple worldwide healthcare challenges $(1,2)$. As of April 2020, more than 1,500,000 patients have been reported, while the death toll has exceeded 100,000 . Although lungs are the most commonly affected organs, involvement of kidney, liver, coagulation system etc. has been reported (3-5). It is well known that certain viruses related to respiratory and gastrointestinal infections can cause myocarditis. Therefore, concerns of SARS-CoV-2 infection associated cardiac injury, and cardiac specific mortality, have emerged (6). It was reported that hypersensitive cardiac troponin I (hs-c TnI) increased significantly in $12 \%$ and $23 \%$ the patients with COVID-19 $(3,7)$. However, the small sample sizes limit the generalizability of these findings. The true impact of SARS$\mathrm{CoV}-2$ on the heart, especially in the area where medical resources were under less pressure than high risk areas over the same period remains largely unclear.

Zhuhai city, as one of the special economic zones in China, is located in south-central Guangdong province. As the only designated hospital of COVID-19 in Zhuhai, we started receiving COVID-19 patients on January 17th. As of March 10th, all the patients were cured except for one old male patient died from COVID-19 though extracorporeal membrane oxygenation (ECMO) treatment had been used. Patients were all imported cases from high risk areas, or cases who had close contact with them. We retrospectively analyzed demographic, clinical, laboratory and cardiovascular imaging data of all consecutive COVID-19 patients admitted into our hospital from January 17th, 2020 to February 18th, 2020, to determine the association of viral infection with acute myocardial injury.

We present the following article in accordance with the STROBE reporting checklist (available at http://dx.doi. org/10.21037/cdt-20-607).

\section{Methods}

The study conforms to the ethical guidelines of the Declaration of Helsinki(as revised in 2013) and was approved by the institutional research board of the Human Subjects Committee of Fifth Affiliated Hospital of Sun Yat-sen University and registered with the official website of China Clinical Trial Registration Center (ChiCTR2000030952). Written informed consent was waived, because it was a retrospective observational study.

Clinical data of all confirmed COVID-19 cases older than 14 years old admitted into Fifth Affiliated Hospital of Sun Yat-sen University from January 17th, 2020 to February 18th, 2020 were collected. The diagnosis and clinical severity classification of COVID-19 were based on the seventh edition guideline released by the Chinese National Health Commission. Criteria of clinical severity classification were as follows: (I) minimal. Mild clinical symptoms without pneumonia in imaging; (II) common. Fever, respiratory tract and other symptoms with pneumonia in imaging; (III) severe. Defined by if there are any of the following conditions. (I) Dyspnea, respiratory rate $\geq 30$ per min, (II) resting hypoxia $\mathrm{SaO}_{2} \leq 93 \%$, (III) $\mathrm{PaO}_{2} / \mathrm{FiO}_{2} \leq 300 \mathrm{mmHg}$. 4.Critical. Defined by if there was one or more of the following conditions. Respiratory failure requiring mechanical ventilation, shock or other organ failure requiring ICU care (8). Cardiac injury was diagnosed if there was increase in serum levels of specific cardiac biomarkers, e.g., hs-cTnI, creatine kinase MB (CK-MB) $(3,9)$.

The normal range of hs-c TnI was $0-0.010 \mu \mathrm{g} / \mathrm{L}$. The normal range of CK-MB was 0-25 U/L. In this study, elevated hs-cTnI referred to above $0.0229 \mu \mathrm{g} / \mathrm{L}$, the 99 th percentile of upper reference limit. Elevated CK-MB was defined as a level twice higher than upper reference limit. Total severity score (TSS) based on CT image was a semiquantitative scoring system used to quantitatively estimate the pulmonary involvement of ground glass opacity, crazypaving pattern, and consolidation resulted from viral pneumonia. Each of the 5 lung lobes was visually scored from 0 to 5 as: 0 , no involvement; $1,<5 \%$ involvement; 2, 25\% involvement; 3, 26-49\% involvement; 4, 50-75\% involvement; $5,>75 \%$ involvement. The total CT score was the sum of the individual lobar scores and ranged from 0 (no involvement) to 25 (maximum involvement) $(10,11)$.

\section{Statistical analysis}

Continuous variables were expressed as median (IQR) and compared with the Mann-Whitney U test; categorical variables were expressed as number (\%) and compared by $\chi^{2}$ test or Fisher's exact test between cardiac injury and non-cardiac injury groups. A two-sided $\alpha$ of less than 0.05 was considered statistically significant. Factors with a possible influence on cardiac injury were calculated using the logistic regression model (univariate and multivariate). The number of covariables entered into the multivariate 
model was limited due to the number of cardiac injury events. The variables with $\mathrm{P}$ value $<0.05$ were in the final model. Statistical analyses were done using SPSS 22.0 for Windows.

\section{Results}

A total of 93 patients were included in the study. Hs-cTnI or CK-MB was measured in all of them. The median number of times that hs-cTnI was tested during the hospitalization was 13 in the group with cardiac injury and 5 in the patients without cardiac injury. A total of $20(21.5 \%)$ patients had received transthoracic echocardiography and 27 (29.0\%) had received electrocardiogram at the time when cardiac injury was suspected. Cardiac injury was observed in $9(9.7 \%)$ patients and the median level of hs-c TnI in cardiac injury group was $0.085 \mu \mathrm{g} / \mathrm{L}$ (IQR $0.027-0.560 \mu \mathrm{g} / \mathrm{L}$ ). None of the patients had experienced fulminant myocarditis or cardiogenic shock.

\section{Demographic and clinical characteristics}

Demographic and clinical characteristics of the 93 included patients were showed in Table 1 . The mean age was 48.0 years (IQR 35.5-62.5). 41 (44.1\%) patients were male and $52(55.9 \%)$ were female. Minimal, common, severe, and critical cases accounted for $18.3 \%, 58.1 \%, 17.2 \%$ and $6.5 \%$ respectively. As of March 10th, all the patients were cured except for one old male patient died from COVID-19 though aggressive therapy including ECMO had been used.

As compared with patients without cardiac injury, the median age of patients with cardiac injury were significantly older (65.0 vs. 44.0, $\mathrm{P}<0.05$ ), combining hypertension was significantly more common $(44.4 \%$ vs. $14.3 \%, \mathrm{P}<0.05)$, and the proportion of severe -critical cases among them were greater $(77.8 \%$ vs. $17.9 \%, \mathrm{P}<0.05)$. On the other hand, there was no significant difference of sex distribution, patient type, diabetes, hyperlipemia, cardiovascular disease, chronic lung disease and malignancy between the two groups. As to heart-related symptoms, fatigue was more common in patients with cardiac injury than in patients without cardiac injury $(44.4 \%$ vs. $6.0 \%, \mathrm{P}<0.05)$ while chest pain was not statistically different between the two groups.

\section{Laboratory findings}

The laboratory parameters of the enrolled patients during critical phase were compared between non-cardiac injury group and cardiac injury group (Table 2). Based on our statistical analysis, patients with cardiac injury had higher neutrophil count $\left(5.1 \times 10^{9} / \mathrm{L}\right.$ vs. $\left.3.0 \times 10^{9} / \mathrm{L}, \mathrm{P}<0.05\right)$ and C-reactive protein level $(45.8$ vs. $9.9 \mathrm{mg} / \mathrm{L}, \mathrm{P}<0.05)$ but lower lymphocyte count $\left(0.7 \times 10^{9} / \mathrm{L}\right.$ vs. $\left.1.2 \times 10^{9} / \mathrm{L}, \mathrm{P}<0.05\right)$ and serum albumin level (32.7 vs. $37.0 \mathrm{~g} / \mathrm{L}, \mathrm{P}<0.05)$ than patients without cardiac injury. Patients with cardiac injury were more likely have elevation of $\mathrm{N}$-terminal proBNP (NT-proBNP) in comparison (66.7\% vs. $10.0 \%$, $\mathrm{P}<0.05)$. The median TSS score between the two groups was significantly different, the cardiac injury group was 8.0 points and the non-cardiac injury group was 3.0 points by contrast.

\section{Echocardiography parameters}

Twenty $(21.5 \%)$ patients had underwent transthoracic echocardiography at the time when cardiac injury was suspected. Echocardiography parameters of the two groups had also been compared (Table 3). Left atrial diameter (LAD) in cardiac injury group was larger than that in non-cardiac injury group (38.0 vs. $35.0 \mathrm{~mm}, \mathrm{P}<0.05)$. No statistical difference of left ventricular end-diastolic diameter (LVEDD), interventricular septal diameter (IVSd), left ventricle posterior wall (LVPW), left ventricular ejection fraction (LVEF), E/A ratio, pulmonary artery systolic pressure (PASP) and right ventricle end-diastolic diameter (RVDd) was found between the two groups.

\section{Cardiac magnetic resonance imaging data of 3 patients with cardiac injury}

Furthermore, cardiac magnetic resonance (CMR) imaging was performed on the 3 critical patients with increased hs-cTnI. One case was a 65 -year-old male with smoking history and surgical history for atrial myxoma and bladder cancer. He once complained of chest tightness for one day during hospitalization. His hs-cTnI value was the highest among all the patients, reaching $2.6 \mu \mathrm{g} / \mathrm{L}$. CMR at the $32 \mathrm{nd}$ day after he underwent hs-c TnI elevation showed subacute myocardial infarction in the inferior wall (Figure 1). Then coronary computed tomography angiography (cCTA) of him demonstrated a severe stenosis at the middle of right coronary artery. The second case was a 71-year-old female with a history of hypertension and hyperlipemia. She did not complain of chest pain or palpitation and her highest hs-cTnI during hospitalization was $0.056 \mu \mathrm{g} / \mathrm{L}$. CMR at the 52nd day after he underwent hs-cTnI elevation showed a 
Table 1 Demographics and baseline characteristics of COVID-19 patients

\begin{tabular}{|c|c|c|c|c|}
\hline Characteristics & All patients $(n=93)$ & Cardiac injury $(n=9)$ & Non-cardiac injury $(n=84)$ & $P$ value \\
\hline Sex & & & & 0.279 \\
\hline Male & $41(44.1)$ & $6(66.7)$ & $35(41.7)$ & \\
\hline Female & $52(55.9)$ & $3(33.3)$ & 49 (58.3) & \\
\hline Imported case* & $76(81.7)$ & $7(77.8)$ & $69(82.1)$ & \\
\hline Close contact ${ }^{*}$ & $17(18.3)$ & $2(22.2)$ & $15(17.9)$ & \\
\hline \multicolumn{5}{|l|}{ Any comorbidity } \\
\hline Hypertension & $16(17.2)$ & $4(44.4)$ & $12(14.3)$ & 0.044 \\
\hline Cardiovascular disease & $1(1.1)$ & $0(0.0)$ & $1(1.2)$ & 1.000 \\
\hline Chronic lung disease & $3(3.2)$ & $1(11.1)$ & $2(2.4)$ & 0.266 \\
\hline Malignancy & $5(5.4)$ & $2(22.2)$ & $3(3.6)$ & 0.072 \\
\hline Severity & & & & $<0.001$ \\
\hline Minimal & 17 (18.3) & $0(0.0)$ & $17(20.2)$ & \\
\hline Common & $54(58.1)$ & $2(22.2)$ & $52(61.9)$ & \\
\hline Severe & $16(17.2)$ & $3(33.3)$ & $13(15.5)$ & \\
\hline Critical & $6(6.5)$ & $4(44.4)$ & $2(2.4)$ & \\
\hline \multicolumn{5}{|l|}{ Signs and symptoms } \\
\hline Hemoptysis & $2(2.2)$ & $0(0.0)$ & $2(2.4)$ & 1.000 \\
\hline Sore throat & $11(11.8)$ & 1 (11.1) & $10(11.9)$ & 1.000 \\
\hline Catarrh & $11(11.8)$ & $1(11.1)$ & $10(11.9)$ & 1.000 \\
\hline Diarrhea & $5(5.4)$ & $0(0.0)$ & $5(6.0)$ & 1.000 \\
\hline Headache & $7(7.5)$ & 2 (22.2) & $5(6.0)$ & 0.136 \\
\hline Chest pain & $5(5.4)$ & $1(11.1)$ & $4(4.8)$ & 0.406 \\
\hline Fatigue & $9(9.7)$ & $4(44.4)$ & $5(6.0)$ & 0.004 \\
\hline Myalgia & $10(10.8)$ & 2 (22.2) & $8(9.5)$ & 0.248 \\
\hline
\end{tabular}

Table 1 (continued) 
Table 1 (continued)

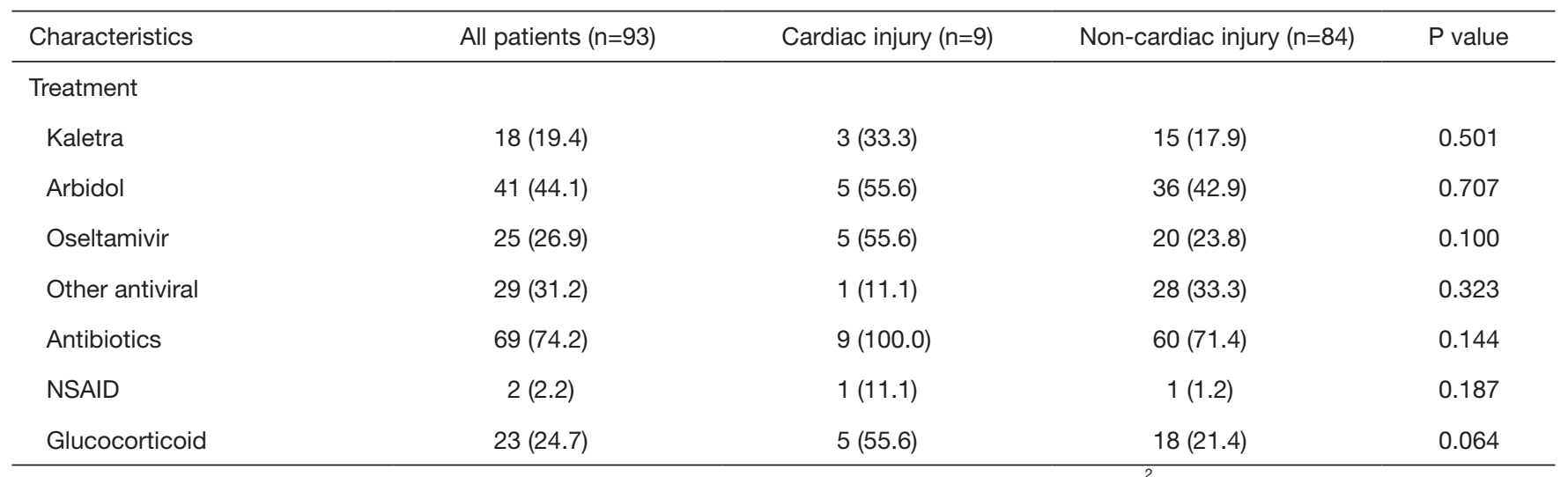

Data are median (IQR) or $\mathrm{n}(\%)$. $\mathrm{P}$ values comparing cardiac injury and non-cardiac injury are from $\chi^{2}$ test, Fisher's exact test, or MannWhitney U test. ${ }^{*}$ Imported case: Imported case of COVID-19 from high risk areas. ${ }^{*}$ Close contact: Close contact with imported case. NSAID, nonsteroidal anti-inflammatory drug.

Table 2 Laboratory findings of COVID-19 patients during critical phase

\begin{tabular}{|c|c|c|c|c|}
\hline Laboratory findings & All patients $(n=93)$ & Cardiac injury $(n=9)$ & Non-cardiac injury $(n=84)$ & $P$ value \\
\hline Times of cTnl test & $6(4-8)$ & $13(11-16)$ & $5(3-7)$ & $<0.001$ \\
\hline CK-MB, U/L & $13.4(10.1-15.9)$ & $13.6(9.4-16.0)$ & $13.3(10.2-16.0)$ & 0.984 \\
\hline White blood cell count, $\times 10^{9} / \mathrm{L}$ & $4.8(3.8-6.2)$ & $6.8(4.4-7.7)$ & $4.6(3.8-5.8)$ & 0.058 \\
\hline Lymphocyte count, $\times 10^{9} / \mathrm{L}$ & $1.1(0.8-1.5)$ & $0.7(0.3-0.8)$ & $1.2(0.8-1.5)$ & 0.002 \\
\hline Creatinine, $\mu \mathrm{mol} / \mathrm{L}$ & $73.1(62.5-86.7)$ & $86.0(69.0-94.7)$ & $71.8(62.1-86.3)$ & 0.085 \\
\hline Albumin, g/L & 36.7 (35.3-38.4) & $32.7(31.3-37.1)$ & $37.0(35.5-38.5)$ & 0.007 \\
\hline C-reactive protein, $\mathrm{mg} / \mathrm{L}$ & $11.4(2.7-36.7)$ & $45.8(16.8-155.5)$ & $9.9(2.2-30.7)$ & 0.010 \\
\hline TSS score & $3.0(1.0-6.0)$ & $8.0(4.0-10.5)$ & $3.0(1.0-6.0)$ & 0.023 \\
\hline
\end{tabular}

Data are median (IQR) or $\mathrm{n}(\%)$. $\mathrm{P}$ values comparing cardiac injury and non-cardiac injury are from $\chi^{2}$ test, Fisher's exact test, or MannWhitney U test. Hs-cTnl, hypersensitive cardiac troponin I; CK-MB, creatine kinase-MB; NT-proBNP, N-terminal-pro-brain natriuretic peptide; TSS score, total severity score.

dilated left ventricle, regional wall motion abnormality and ventricular aneurysm in the apex. Her cCTA demonstrated chronic occlusion in the proximal segment of the left anterior descending artery. Thus, ischemic cardiomyopathy was diagnosed. The third case was a 75-year-old female. She had no history of cardiovascular disease but hypertension. CMR at the 45th day after she underwent cTnI elevation revealed asymmetrical hypertrophy of the left ventricular wall and focal late gadolinium enhancement in the lower lateral wall of left ventricular basement segment. Her interventricular septum was $16.2 \mathrm{~mm}$ thick while the left ventricular posterior wall was $8.5 \mathrm{~mm}$.

\section{Risk factors of cardiac injury}

Univariate logistic regression analysis showed that being 
Table 3 Transthoracic echocardiography parameters of COVID-19 patients during critical phase

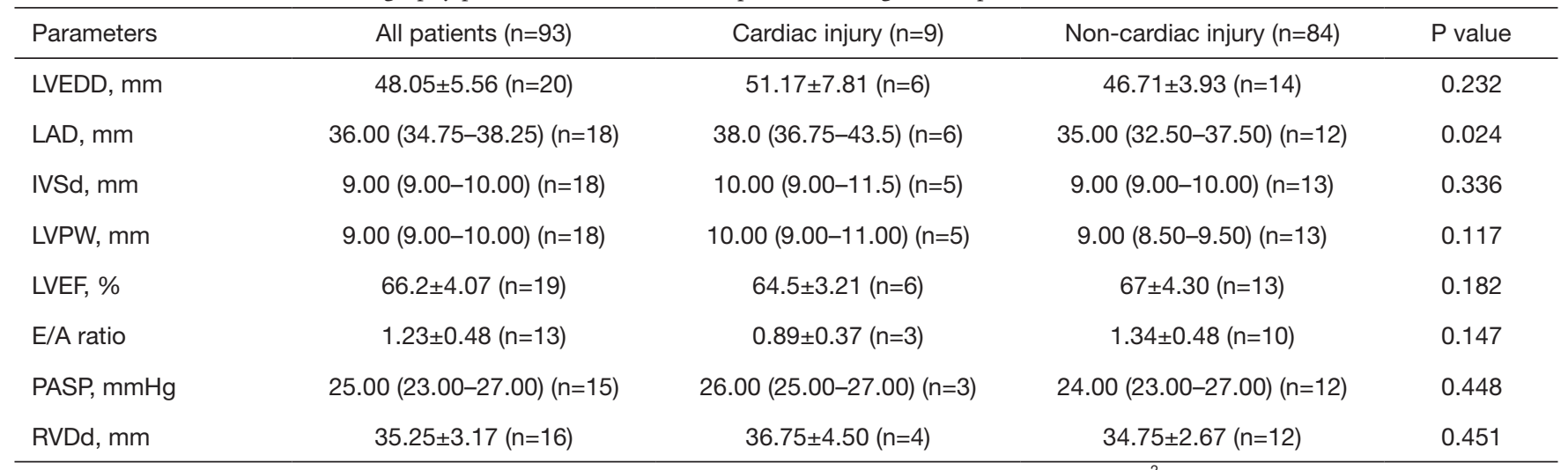

Data are median (IQR) or $\mathrm{n}(\%)$. $\mathrm{P}$ values comparing cardiac injury and non-cardiac injury are from $\chi^{2}$ test, Fisher's exact test, or MannWhitney U test. LVEDD, left ventricular end-diastolic diameter; LAD, diameter; IVSd, interventricular septal diameter; LVPW, left ventricle posterior wall; LVEF, left ventricular ejection fraction; E/A ratio, the ratio of mitral peak velocity of early filling (E) to late filling (A); PASP, pulmonary artery systolic pressure; RVDd, right ventricle end-diastolic diameter.
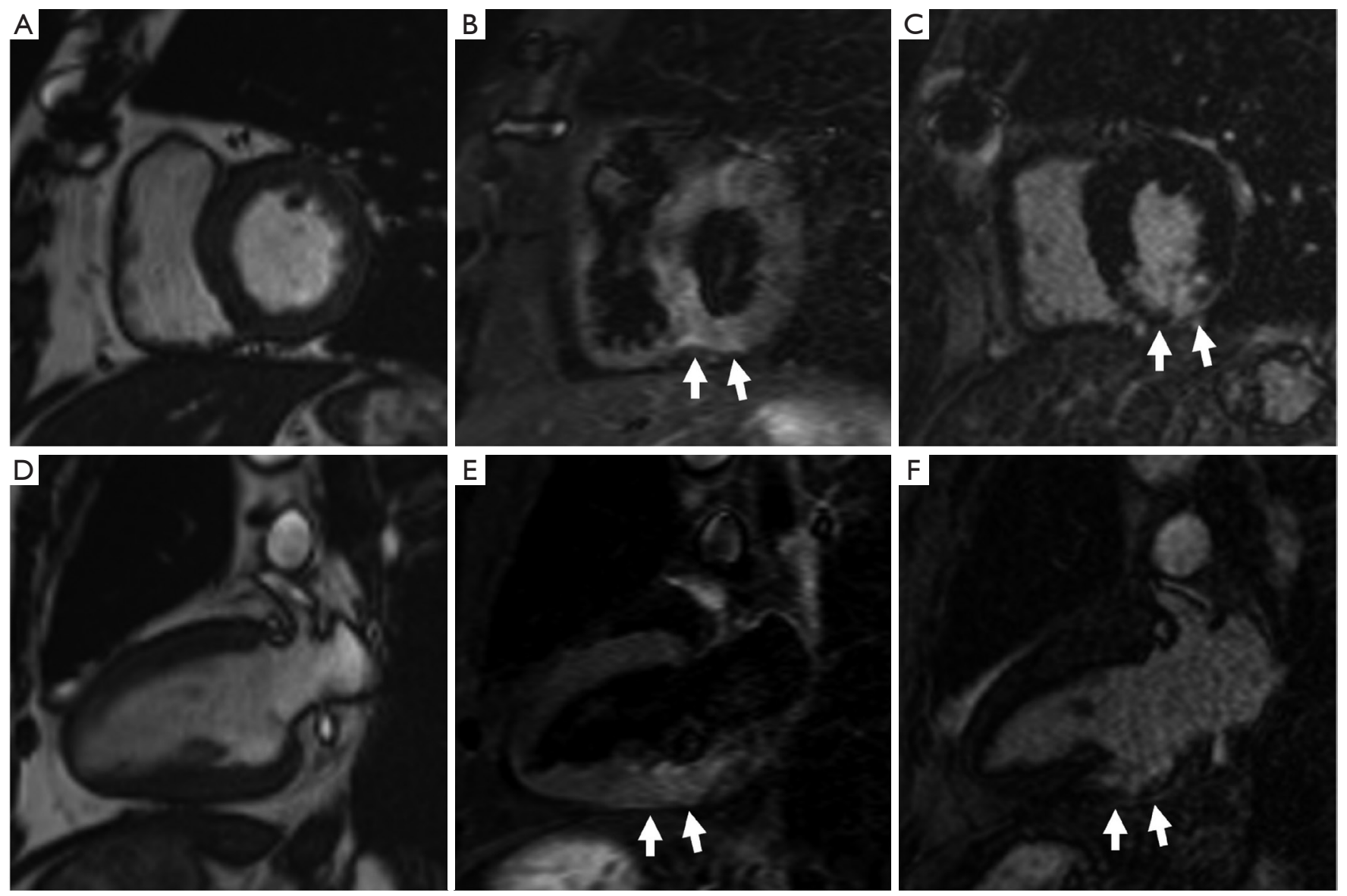

Figure 1 The cardiac magnetic resonance images of one male COVID-19 patient with cardiac injury. (A,B,C) Short-axis; (D,E,F) long-axis. (A,D) Cine-SSFP images show absence of hypertrophy of the left ventricular wall; (B,E) T2 weighted images show myocardial edema of the inferior and inferoseptal walls (white arrows); (C,D) T1-weightedimages show transmural late gadolinium enhancement of the inferior and inferoseptal wall (white arrows), suggestive of myocardial infarction. 
Table 4 Univariate and multivariate logistic regression analysis for cardiac injury

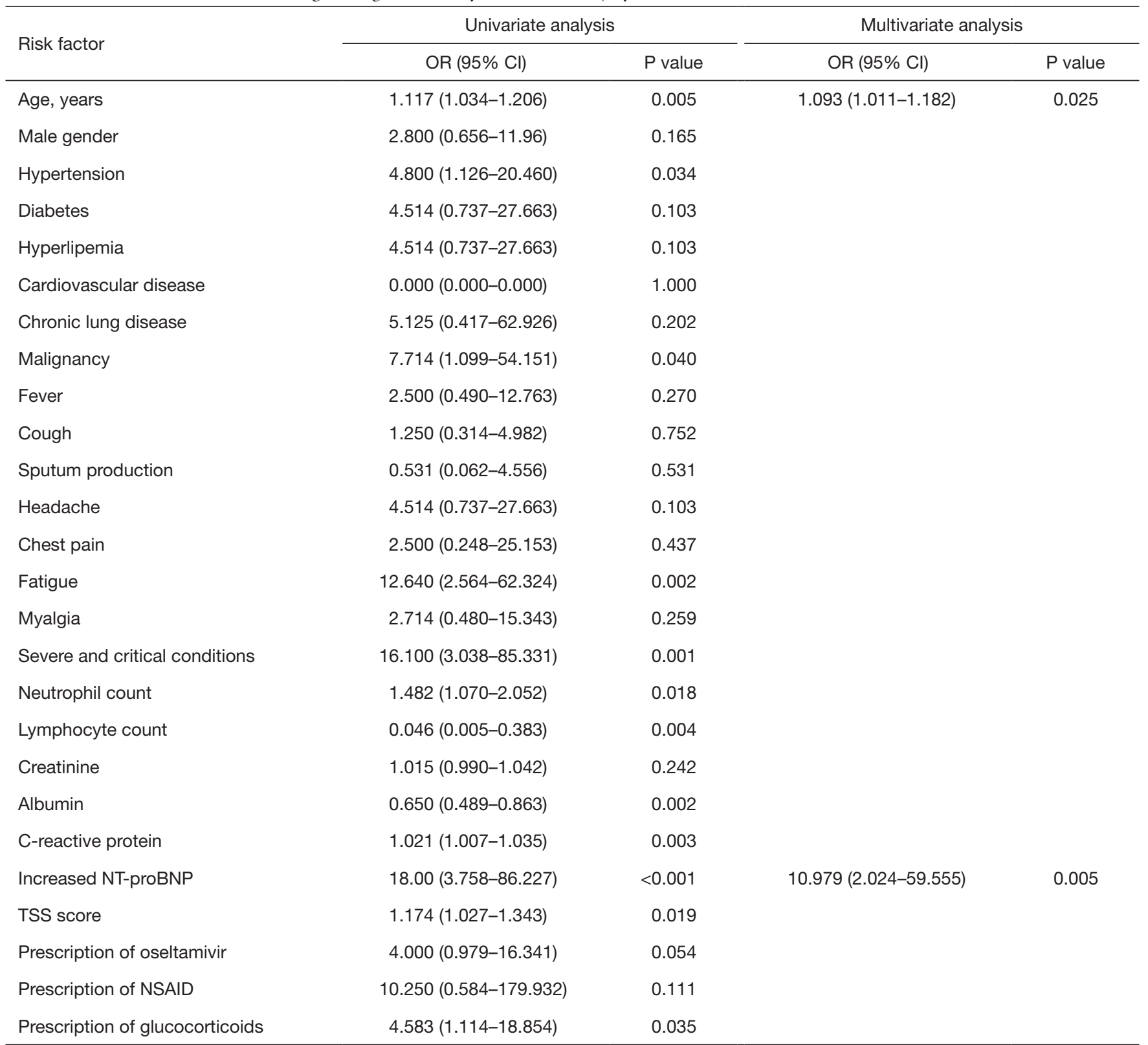

Chronic lung disease includes chronic obstructive pulmonary disease and tuberculosis. TSS, total severity score; NSAID, nonsteroidal anti-inflammatory drug.

older, combining hypertension, malignancy, symptom of fatigue, severe and critical COVID-19, higher neutrophil count, lower lymphocyte count, lower albumin level, higher C-reactive protein level, increased NT-proBNP, higher TSS score, prescription of Oseltamivir and glucocorticoids were associated with cardiac injury. In multivariable logistic regression analysis, older age (OR: 1.093, 95\% CI: $1.011-$
1.182) and increased NT-proBNP (OR: $10.979,95 \%$ CI: 2.024-59.555) were risk factors for cardiac injury (Table 4).

\section{Discussion}

Our study focused on acute cardiac injury in COVID-9 patients in Zhuhai, a city where medical resources were 
under less pressure than that in high risk areas. The present study showed that acute cardiac injury was seen in the minority of patients presenting with COVID-19, occurring in $9.7 \%$. Evidence for acute myocardial injury was less frequent in our patients compared to that reported from New York City including nearly 3,000 patients with a prevalence of $36 \%$ (12), and that reported from high risk areas in China (ranging from $12 \%$ to $27.8 \%)(3,7,13,14)$. However, a study from Shenzhen (15), Guangdong Province, a city near Zhuhai, reported the prevalence of acute cardiac injury was $8.4 \%$, which was quite close to our finding. Similar to these reports, we also demonstrated that patients with cardiac injury tended to be older and were more likely to combine with hypertension, more likely to be categorized as severe-critical cases. The reason why acute cardiac injury was less frequent in our patients than high risk areas in China and New York might be related to reality that our patients was not that sick as patients in those areas, since our minimal, common, severe, and critical cases accounted for $18.3 \%, 58.1 \%, 17.2 \%$ and $6.5 \%$ respectively and the median TSS score, a semi-quantitative score based on CT image to quantitatively estimate the severity the pulmonary involvement resulted from viral pneumonia, of our patients was 3 . It is quite frankly probable that in a sicker population with more lung involvement and more hypoxemia that elevated hs-c TnI would be more prevalent. Besides, as New York or high risk areas in China at that time had much more COVID-19 cases than Guangdong Province, the regional health care systems of them were faced with much more challenge, which might have potentially increased risk of cardiac injury due to delayed hospital admission. Another finding of our study was that elevation of cardiac injury markers of in our cohort was often mild. This finding was in keeping with the report from New York City (12).

Our multivariable logistic regression analysis showed that older age and increased NT-proBNP was significantly associated with cardiac injury in COVID-19 patients. Elder patients are more likely to have preceding cardiovascular comorbidities, it is easy to understand why older age was associated with cardiac injury in COVID-19. When it came to the association between NT-proBNP and cardiac injury in COVID-19, as echocardiography of our patients with cardiac injury showed preserved ejection fraction, enlarged left atrium and all their renal function was normal, whether there was volume overload should be firstly evaluated. However, our patients had not received any excessive rehydration. Then we inferred it might be the right heart dysfunction resulted from acute respiratory distress syndrome (ARDS) due to the virus infection that caused NT-proBNP and hs-cTnI increasing simultaneously, as it was quite commonly reported in ARDS (16).

Myocarditis theoretically maybe another explanation for increased hs-cTnI and NT-proBNP, since acute myocarditis related to preceding respiratory tract infections is often a matter of concern. Previously, it was reported that acute myocarditis and heart failure could be observed in patients infected with Middle Eastern Respiratory Syndrome coronavirus (MERS-CoV) while elevation of cardiac injury markers in patients with Severe Acute Respiratory Syndrome coronavirus (SARS-CoV) is not obvious $(17,18)$. Considered as a new human-infecting beta coronavirus, SARS-CoV-2 shares $79 \%$ identity with the SARS-CoV and 50\% identity with MERS-CoV $(2,19)$. Life-threatening cardiac tamponade complicating myopericarditis in COVID-19 patients has been described (20), while a case report of endomyocardial biopsy of COVID-19 present with cardiogenic shock clinically mimicking fulminant myocarditis demonstrated low-grade myocardial inflammation and absence of myocyte necrosis. The first pathologic anatomy of a patient with confirmed COVID-19 death mentioned that there were a few interstitial mononuclear inflammatory infiltrates but no other substantial damage in the heart tissue $(21,22)$. As our three MRI studies were not performed at the acute phrase of cardiac injury, it is possible that evidence of myocarditis that may have been present early on would have resolved by the time when we performed these MRI studies. Based on our research, we did not have enough data to rule out cardiac injury caused by myocarditis. Nevertheless, it was worth noticing that CMR on our three COVID-19 patients with cardiac injury demonstrated one acute myocardial infarction, one ischemic cardiomyopathy, and the other one was left ventricular hypertrophy, suggesting cardiac injury in those patients might be related to their preexisting cardiovascular diseases. Acute respiratory dysfunction and hypoxemia caused by COVID-19 could exacerbate myocardial oxygen supply-demand imbalance in patients with underlying cardiovascular diseases. Consequently, type 2 myocardial infarction took place.

By reviewing recent literatures, in addition to the putative mechanisms mentioned above, we can learn that SARS-CoV-2 could also induce cardiac injury through the following ways: first, SARS-CoV-2 is thought to infect host cells through angiotensin-converting enzyme 2 (ACE2) and ACE2 is widely expressed in arterial and venous 
endothelial cells, which can cause direct viral injury by either direct viral entry into myocardial endothelial cells or indirect immunological response (23). Second, SARS$\mathrm{CoV}-2$ infection can trigger a cytokine storm which can also cause heart injury (24). Besides, emerging evidence indicates that endothelium cell dysfunction is a central feature of COVID-19. As the vascular endothelium forms a critical interface between the circulatory system and is the key driver of cytokine dysregulation in ARDS as well as multiple cardiovascular pathologies, endothelium injury of the coronary arteries may play a critical role in COVID-19 related cardiac injury (25).

To date, there has been limited published data on CMR in COVID-19 patients. Here we present CMR characteristics of three patients with significantly increased hs-cTnI level, suggesting that myocardial infarction or cardiac injury secondary to respiratory dysfunction and hypoxemia should always be kept in mind. Therefore, we advise that, when caring for severe-critical COVID-19 patients with cardiac injury, we should pay more attention to improving respiratory dysfunction and hypoxemia and finding out the underlying cardiovascular comorbidities.

Our study has several limitations: First, most of the patients did not undergo electrocardiogram or echocardiography. Whereas those were minimal or common cases, and their hs-cTnI and/or CK-MB level were normal, they did not complain of chest pain, shortness of breath, or palpitations, so the likelihood of cardiac injury among them was low. Second, the three MRI studies were not performed at the acute phrase of cardiac injury.

\section{Conclusions}

In summary, acute cardiac injury was seen in the minority of hospitalized COVID-19 patients in Zhuhai, China. Older age and increased NT-proBNP were associated with acute cardiac injury.

\section{Acknowledgments}

We thank Professor Zhonghe Li, Dr. Zuoquan Zhang, Dr. Cunxue Pan and Dr. Kunwei Li for their help in the clinical severity classification and CT/CMR evaluation of the patients.

Funding: None.

\section{Footnote}

Reporting Checklist: The authors have completed the
STROBE reporting checklist. Available at http://dx.doi. org/10.21037/cdt-20-607

Data Sharing Statement: Available at http://dx.doi. org/10.21037/cdt-20-607

Peer Review File: Available at http://dx.doi.org/10.21037/ cdt-20-607

Conflicts of Interest: All authors have completed the ICMJE uniform disclosure form (available at http://dx.doi. org/10.21037/cdt-20-607). All authors have no conflicts of interest to declare.

Ethical Statement: The authors are accountable for all aspects of the work in ensuring that questions related to the accuracy or integrity of any part of the work are appropriately investigated and resolved. The study conforms to the ethical guidelines of the Declaration of Helsinki (as revised in 2013) and was approved by the institutional research board of the Human Subjects Committee of Fifth Affiliated Hospital of Sun Yat-sen University and registered with the official website of China Clinical Trial Registration Center (ChiCTR2000030952). Written informed consent was waived, because it was a retrospective observational study.

Open Access Statement: This is an Open Access article distributed in accordance with the Creative Commons Attribution-NonCommercial-NoDerivs 4.0 International License (CC BY-NC-ND 4.0), which permits the noncommercial replication and distribution of the article with the strict proviso that no changes or edits are made and the original work is properly cited (including links to both the formal publication through the relevant DOI and the license). See: https://creativecommons.org/licenses/by-nc-nd/4.0/.

\section{References}

1. Li Q, Guan X, Wu P, et al. Early Transmission Dynamics in Wuhan, China, of Novel Coronavirus-Infected Pneumonia. N Engl J Med 2020;382:1199-207.

2. Zhu N, Zhang D, Wang W, et al. A Novel Coronavirus from Patients with Pneumonia in China, 2019. N Engl J Med 2020;382:727-33.

3. Huang C, Wang Y, Li X, et al. Clinical features of patients infected with 2019 novel coronavirus in Wuhan, China. Lancet 2020;395:497-506. 
4. Liu C, Jiang ZC, Shao CX, et al. [Preliminary study of the relationship between novel coronavirus pneumonia and liver function damage: a multicenter study]. Zhonghua Gan Zang Bing Za Zhi 2020;28:148-52.

5. Tang N, Li D, Wang X, et al. Abnormal Coagulation parameters are associated with poor prognosis in patients with novel coronavirus pneumonia. J Thromb Haemost 2020;18:844-7.

6. Inciardi RM, Lupi L, Zaccone G, et al. Cardiac Involvement in a Patient With Coronavirus Disease 2019 (COVID-19). JAMA Cardiol 2020;5:819-24.

7. Yang $\mathrm{X}, \mathrm{Yu} \mathrm{Y}, \mathrm{Xu} \mathrm{J}$, et al. Clinical course and outcomes of critically ill patients with SARS-CoV-2 pneumonia in Wuhan, China: a single-centered, retrospective, observational study. Lancet Respir Med 2020;8:475-81.

8. General Office of National Health Committee. Notice on the issuance of a program for the diagnosis and treatment of novel coronavirus (2019-nCoV) infected pneumonia (trial revised seventh edition). Available online: http:// www.nhc.gov.cn/yzygj/s7653p/202003/46c9294a7dfe4cef8 0dc7f5912eb1989.shtml.

9. Gao C, Wang Y, Gu X, et al. Association Between Cardiac Injury and Mortality in Hospitalized Patients Infected With Avian Influenza A (H7N9) Virus. Crit Care Med 2020;48:451-8.

10. Chang YC, Yu CJ, Chang SC, et al. Pulmonary sequelae in convalescent patients after severe acute respiratory syndrome: evaluation with thin-section CT. Radiology 2005;236:1067-75.

11. Pan F, Ye T, Sun P, et al. Time Course of Lung Changes On Chest CT During Recovery From 2019 Novel Coronavirus (COVID-19) Pneumonia. Radiology 2020;295:715-21.

12. Lala A, Johnson KW, Januzzi JL, et al. Prevalence and Impact of Myocardial Injury in Patients Hospitalized With COVID-19 Infection. J Am Coll Cardiol 2020;76:533-46.

13. Guo T, Fan Y, Chen M, et al. Cardiovascular Implications of Fatal Outcomes of Patients With Coronavirus Disease 2019 (COVID-19). JAMA Cardiol 2020;5:811-8.

14. Shi S, Qin M, Shen B, et al. Association of Cardiac Injury

Cite this article as: Jin L, Tang W, Song L, Luo L, Zhou Z, Fan X, Zhang J, Wu N, Liu K, Chen J. Acute cardiac injury in adult hospitalized COVID-19 patients in Zhuhai, China. Cardiovasc Diagn Ther 2020;10(5):1303-1312. doi: 10.21037/ cdt-20-607
With Mortality in Hospitalized Patients With COVID-19 in Wuhan, China. JAMA Cardiol 2020;5:802-10.

15. Zeng JH, Wu WB, Qu JX, et al. Cardiac manifestations of COVID-19 in Shenzhen, China. Infection 2020:1-10. [Epub ahead of print].

16. Park BH, Kim YS, Chang J, et al. N-terminal probrain natriuretic peptide as a marker of right ventricular dysfunction after open-lung approach in patients with acute lung injury/acute respiratory distress syndrome. J Crit Care 2011;26:241-8.

17. Alhogbani T. Acute myocarditis associated with novel Middle east respiratory syndrome coronavirus. Ann Saudi Med 2016;36:78-80.

18. Chen M, Huo Y, Huo N, et al. [Cardiac presentations in patients with SARS]. Beijing Da Xue Xue Bao Yi Xue Ban 2003;35:556-7.

19. Lu R, Zhao X, Li J, et al. Genomic characterisation and epidemiology of 2019 novel coronavirus: implications for virus origins and receptor binding. Lancet 2020;395:565-74.

20. Hua A, O'Gallagher K, Sado D, et al. Life-threatening cardiac tamponade complicating myo-pericarditis in COVID-19. Eur Heart J 2020;41:2130.

21. Xu Z, Shi L, Wang Y, et al. Pathological findings of COVID-19 associated with acute respiratory distress syndrome. Lancet Respir Med 2020;8:420-2.

22. Tavazzi G, Pellegrini C, Maurelli M, et al. Myocardial localization of coronavirus in COVID-19 cardiogenic shock. Eur J Heart Fail 2020;22:911-5.

23. Iacobazzi D, Baquedano M, Madeddu P, et al. COVID-19, State of the Adult and Pediatric Heart: From Myocardial Injury to Cardiac Effect of Potential Therapeutic Intervention. Front Cardiovasc Med 2020;7:140.

24. Zheng YY, Ma YT, Zhang JY, et al. COVID-19 and the cardiovascular system. Nat Rev Cardiol 2020;17:259-60.

25. Evans PC, Ed Rainger G, Mason JC, et al. Endothelial dysfunction in COVID-19: a position paper of the ESC Working Group for Atherosclerosis and Vascular Biology, and the ESC Council of Basic Cardiovascular Science. Cardiovasc Res 2020. [Epub ahead of print]. 